\title{
Broadband dielectric characterization of novel organic substrates for microwave applications
}

\author{
Muhammad Yusof Ismail1, ${ }^{1,}$, Hasan Ijaz Malik ${ }^{1}$, Sharmiza Adnan ${ }^{2}$ and Shaiful Rizal Masrol ${ }^{1}$ \\ ${ }^{1}$ Universiti Tun Hussein Onn Malaysia (UTHM), Parit Raja 86400, Batu Pahat, Johor, Malaysia \\ ${ }^{2}$ Forest Research Institute Malaysia (FRIM), Jalan Frim, Kepong, 52109 Kuala Lumpur, Selangor, Malaysia
}

\begin{abstract}
This paper presents three novel substrate materials with efficient dielectric characteristics. Different types of innovative substrate materials used for microwave electronics have been discussed. It has been demonstrated that the repeatability of results for paper substrate based electronics can be maintained using the proposed substrate materials. Three different substrate materials derived from recycled sources such as newspaper, copier paper and carton paper and banana pulp have been presented. The paper substrates were manufactured and processed to reduce the moisture content that might degrade the performance. A broadband dielectric material characterization of the proposed substrate shows a stable electromagnetic behaviour over the $\mathrm{X}$ - band operation with relative permittivities of $1.81,1.63$ and 1.84 along with loss tangents of 0.053 , 0.046 and 0.057 respectively.
\end{abstract}

\section{Introduction}

The introduction of innovative materials with potential applications for millimetre and submillimetre frequencies have revolutionized the communication technologies [1][3]. Low - loss dielectric substrate materials with stable electromagnetic behaviour are of great interest for millimetre wave frequencies. Conventional dielectric substrate materials such as epoxy and rubber based substrates include the FR -4 and Roger Duroid substrate materials. They have been widely used in the communication industry for microwave electronic circuit designs. The substrate materials can be grouped in two basic categories depending upon the dielectric permittivity they present i.e. high permittivity material and low permittivity materials. High permittivity materials find applications in electronic circuit designing where no spurious radiations are desired. Low permittivity material are preferred for antenna applications where fringing field are required for radiation purposes.

Sustainable dielectric materials derived from organic sources such as plant sources demonstrate efficient capabilities for antenna applications [4]-[8]. They provide advantages in term of low cost, stable and excellent properties in dry conditions. Different paper substrate materials have been presented for antenna applications. They include commercially available paper materials such as the Mitsubishi Electric photo-paper [4] and the usual paper [5], [6], [9]-[11]. The characterization results of conventional paper differs a lot between the individual runs due to difference of the paper ingredients.
The composition concern is of utter importance since the paper used in the stated works might be from different manufacturers and may consist of different additives. Thus an accurate material characterisation of dielectric is always recommended that could ensure realization of high performance antennas on paper substrate materials.

A substrate material based on aromatic organic material Benzocyclobutene have been proposed for millimetre and sub-millimetre wave frequencies [12]. It has been proposed to address the large dielectric losses of conventional substrate materials at sub-millimetre wave frequencies. The proposed dielectric substrate material has been characterized over a wide frequency range of 11 $-65 \mathrm{GHz}$, where it presents a stable dielectric constant of 2.65 and a loss tangent of $0.001-0.009$ [13].

Dielectric substrates based on textile materials for wearable electronics are also being discussed in [14][17]. These materials provide low dielectric constants between 1-2 due to porous structure and the presence of air. Textile substrates are always having a dynamic equilibrium with humidity and temperature of air of their surroundings. This makes textile materials vulnerable to unstable properties due to changing moisture content. The moisture content absorbed in the textile fibre increases the dielectric constant and loss tangent and it decreases the impedance bandwidth of the antenna [15]. A successful integration of a waveguide antenna on a textile substrate material has been demonstrated at a measured gain of 3.9 $\mathrm{dBi}$ for $2.45 \mathrm{GHz}$ [14]. Thus a further improvement of textile materials in terms of designs and optimization will help enhance the performance and behaviour of textile antennas.

\footnotetext{
Corresponding author: yusofi@uthm.edu.my
} 
This article provides an innovative substrate material based on recycled materials derived from different organic sources such as copier paper, newspaper and the carton paper. It has been demonstrated that the controlled composition of the of different ingredients lead to diverse material properties. The proposed dielectric material is thoroughly characterized over X - band frequency range and the results of material characterization are presented and compared for analysis.

\section{Proposed Dielectric Materials}

Recycled materials derived from sources newspaper, carton paper and the copier paper was processed to manufacture dielectric substrates. The constituents of the proposed paper substrate are depicted in Fig. 1. The proposed dielectric material is based on the combination of two different constituents: the main ingredient and the binding ingredient. Banana fibre is used as a binding ingredient for the paper and it adds tensile strength properties to the paper substrate. The amount of banana pulp depends on the particle size of the main constituent. The composition ratios of the material are presented in Table 1.

Table 1. Physical composition of proposed dielectric materials

\begin{tabular}{|c|c|c|c|c|}
\hline Substrate & $\begin{array}{c}\text { Banana } \\
\text { fibre }\end{array}$ & $\begin{array}{c}\text { News- } \\
\text { paper }\end{array}$ & $\begin{array}{c}\text { Carton } \\
\text { paper }\end{array}$ & $\begin{array}{c}\text { Copier } \\
\text { paper }\end{array}$ \\
\hline RCP50 & 50 & -- & -- & 50 \\
\hline RCR75 & 25 & -- & 75 & -- \\
\hline RNP50 & 50 & 50 & -- & -- \\
\hline
\end{tabular}

A paper material is a suspension of different size particles suspended in a bonding medium. The ratio between the bonding medium and the particles depends upon the individual size characteristics of the particles. In the manufacturing of the paper substrate the banana fibre was added with controlled compositions according to the size of main ingredient. As shown in Table 1 the percentage of banana fibre is $50 \%$ for copier paper (RCP50) and newspaper (RNP50) based substrate materials and it is 25 $\%$ for carton based paper substrate (RCR75). Initially the substrate materials were produced in the form of thin sheets which are not suitable for microwave antenna applications. Thus multiple sheets were combined together to increase the substrate thickness. The thickness of the substrate material plays an important role in the accuracy of dielectric material characterization results. The substrate materials were then passed thoroughly through rolling and pressing stages at temperatures of $30-35^{\circ} \mathrm{C}$ to remove any moisture content. Higher temperatures were avoided during pressing and rolling stages so that paper properties might not be affected.

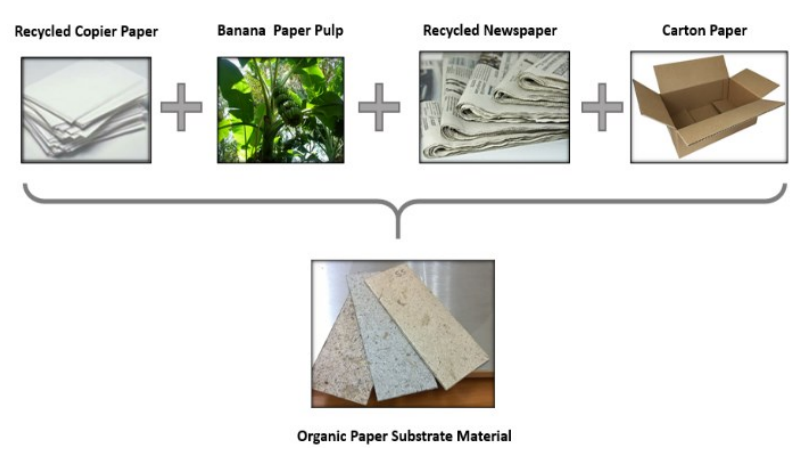

Fig. 1. Different recycled ingredients for paper substrate material

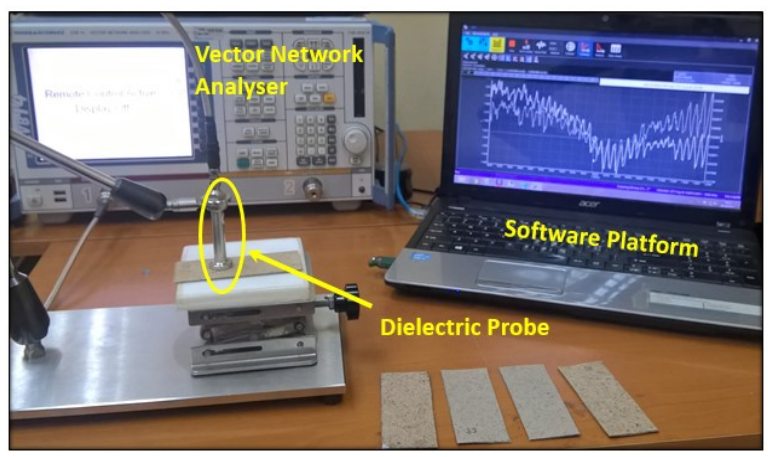

Fig. 2. Dielectric material characterization set-up

\section{Dielectric Material Characterization}

The electrical behaviour and the performance of proposed paper substrates was determined using a broadband characterization technique. A dielectric probe was used for this purpose. The principle of the dielectric probe is based on an open ended coaxial feed line. The fringing fields at the open end of the probe are utilized to estimate the properties of the material held in close proximity. The method is well suited for flat solids, liquids and semisolids. The material under test is held in close contact with the face of the dielectric probe where the fringing fields pass through the material. The velocity of a wave in a medium is dependent to on the dielectric constant of material, using this principle the dielectric behaviour of material under test is estimated. The process can be used for determining the relative dielectric constant and the electrical loss factors of materials.

The complete set-up used for the dielectric material characterization is presented in Fig. 2. The dielectric probe was a DAK $3.5 \mathrm{~mm}$ probe with a broadband frequency analysis range of $200-20 \mathrm{GHz}$. The complete set-up shown in Fig. 1 consists of the probe, a Rodhe \& Schwarz $14 \mathrm{GHz}$ vector network analyser and a software platform installed on a PC. The probe is operated by the software platform via network analyser. Before the material testing the probe is thoroughly calibrated for any errors and discrepancies using air, copper strip and water for open, short and load calibrations. Load calibration was performed using water as load due to universal properties of water. The temperature of water was specially taken in to account due its temperature dependent properties. 


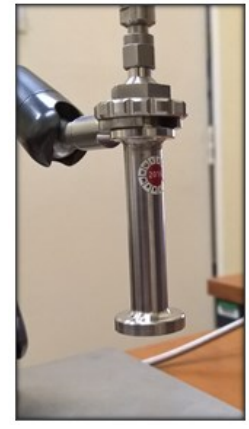

(a)

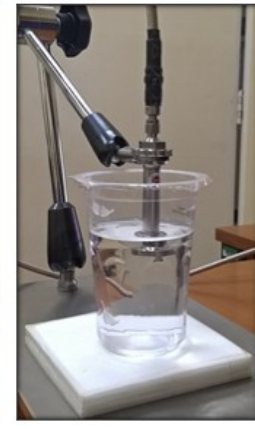

(b)

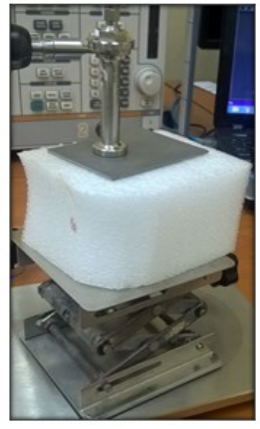

(c)
Fig. 3. Material characterization (a) Dielectric probe (b) Load calibration (c) Material under test

The dielectric probe and load calibrations are presented in Fig. 3. The probe is completely submerged in the water for load calibration as shown in Fig. 3(b). A vertically moveable fixture was used to maintain a firm contact between the probe face and the material under test as shown in Fig. 3(c). A firm contact is necessary for reliable results since any air gap will lead to measurement discrepancies.

Multiple samples were measured to ensure repeatability and reliability of the results. The results of the dielectric material characterization in term of relative dielectric permittivity and the loss tangent are presented in Fig. 4 and Fig. 5. Dielectric permittivity results presented in Fig. 4 show a broadband analysis of proposed paper substrate material over the $\mathrm{X}$ - band frequency range. The presented results show a gentle behaviour of relative permittivity of the material over the entire band of interest with minor deviations. The results show the lowest dielectric permittivity for RCR75 substrate material while highest permittivity results are shown by RNP50 substrate material. Fig. 5 shows the loss tangent results for the proposed substrate materials. The presented curves show a close trend of loss tangent for RNP50 and RCP50 substrate materials. The trend shows a decrease in loss from $8-10 \mathrm{GHz}$ while a constant loss is shown for the range of $10-12 \mathrm{GHz}$. The finding from the Fig. 4 and Fig. 5 are tabulated in Table 2.

Table 2. Dielectric material characterization results

\begin{tabular}{|c|c|c|c|}
\hline Substrate & $\boldsymbol{\varepsilon} \mathbf{r}$ & $\tan \delta$ & $\begin{array}{c}\text { Height } \\
(\mathbf{m m})\end{array}$ \\
\hline RCP50 & 1.81 & 0.053 & 1.45 \\
\hline RCR75 & 1.63 & 0.046 & 1.62 \\
\hline RNP50 & 1.84 & 0.057 & 1.12 \\
\hline
\end{tabular}

The substrate heights of samples for the material used for characterization are also presented in Table 2 . The presented results are the mean values over the $\mathrm{X}-$ band frequency range. The results show a mean relative permittivity of 1.81, 1.63 and 1.84 for RCP50, RCR75 and RNP50 substrate materials. The loss tangent results show a maximum loss tangent of 0.057 for RNP50 substrate

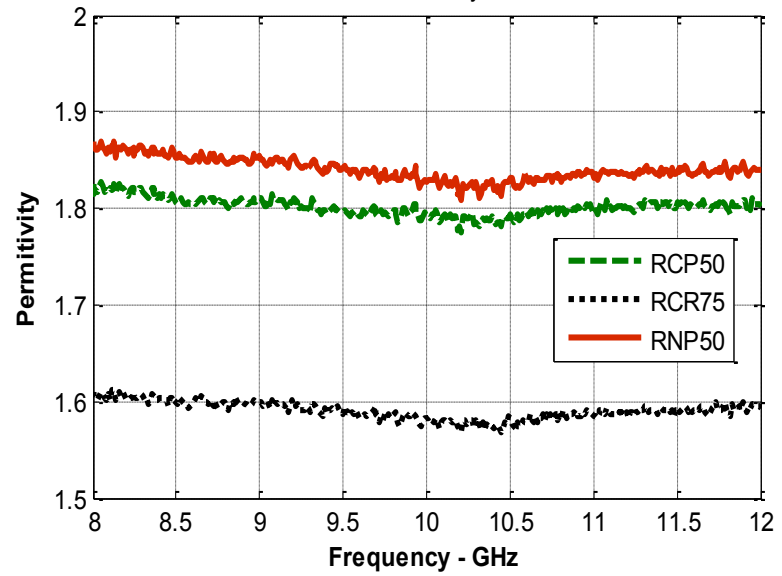

Fig. 4. Relative permittivity results of proposed substrate materials

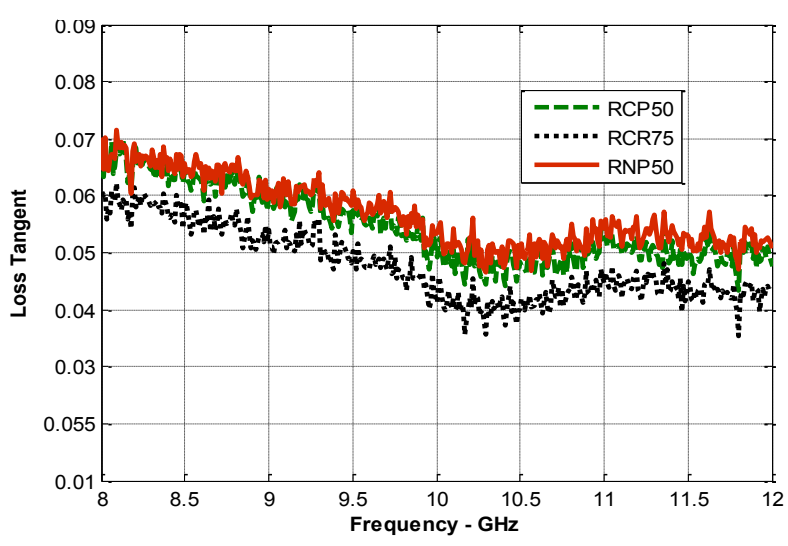

Fig. 5. Loss tangent measurement results for proposed paper substrates

material, while a minimum loss tangent of 0.046 is shown by RCR75. An intermediate loss tangent of 0.053 is presented by RCP50 substrate material.

Material characterization of the proposed substrate material shows that a diverse trend in dielectric properties can be achieve by controlling the composition of organic substrate materials. The proposed substrate materials will provide excellent electromagnetic properties for microstrip patch antenna design. The low permittivity of the material will help to enhance the fringing field effects required for microstrip patch antennas [18]. This will improve the radiation efficiency of the antenna. In order to protect the paper substrate from harsh environmental conditions water proof laminations should be used for microstrip antennas. Since the moisture content may contaminate the substrate resulting in degradation of material properties.

\section{Conclusion}

Dielectric materials find a wide variety of applications in different components such as capacitors, PCBs and microstrip antennas. Researchers have introduced a lot of different types of substrate materials with diverse material properties. The use of paper substrates for low cost, quick printed of electronic components have revolutionized the circuit printing industry. However due to difference in the composition and additives of paper from different 
manufacturers, performance of paper substrate based electronics lack repeatability of results. In order to address the issue this article proposes three different types of paper substrate materials. The composition of the paper substrate is carefully controlled in order to develop a relationship between the material properties and the ingredient of paper. A thorough characterization of paper substrate material show efficient low dielectric material properties with relative permittivities of 1.81, 1.63 and 1.84 along with dissipation factors of $0.053,0.046$ and 0.057 respectively.

The work was funded by GPPS Grant (VOT466), RACE Grant (VOT1119) and Research and Development (R\&D) Fund awarded by Ministry of Higher Education Malaysia.

\section{References}

[1] Y. Malallah et al., 16th Mediterranean Microw. Symp. (MMS), (2016).

[2] K. Nadaud, R. Gillard, E. Fourn, H. W. Gundel, and C. Borderon, Loughborough Ant. \& Propa. Conf., LAPC, November, 214-217, (2014).

[3] F. L. Martínez et al., Proc. 21st IEEE Int. Symp. on Appl. of Ferroelect. \& 11th IEEE Euro. Conf. on Appl. Polar Dielec. IEEE PFM, 1-4. (2012)

[4] F. Alimenti et al., 2015 IEEE MTT-S Int. Micr. Symp., 14. (2015)

[5] T. Leng, X. Huang, K. Chang, J. Chen, M. A. Abdalla, and Z. Hu, IEEE Ant. Wirel. Propag. Lett. 15, 1565-1568, (2016)

[6] B. S. Cook and A. Shamim, IEEE Ant. Wirel. Propag. Lett., 12, 76-79. (2013)

[7] Aastha, A. Kaur, A. S. Dhillon, and E. Sidhu, Int. Conf. on Wirel. Comm., Sig. Proces. and Netw. (WiSPNET), 2090-2093. (2016)

[8] L. Xu and J. L. W. Li, 10th Int. Symp. on Ant., Prop. and EM Theory, 109-112. (2012)

[9] H. AbuTarboush and a. Shamim, IEEE Ant. Wirel. Propa. Lett., 11, 1-1. 2012.

[10] A. Shamim and H. F. Abutarboush, IET Micro. Ant. Propa., 8, 52-56. (2014)

[11] M. Kanagasabai and J. Kizhekke, IET Micro. Ant. Propag. 9. (2015)

[12] S. Costanzo, F. Venneri, A. Borgia, I. Venneri, and G. Di Massa, IET Sci. Meas. Technol., 5, 134, (2011)

[13] S. Costanzo, I. Venneri, G. Massa, and A. Borgia, J. Infr., Mill., Terahertz Waves, 31, 66, (2009)

[14] S. Agneessens, M. Bozzi, R. Moro, and H. Rogier, Electron. Lett., 48, 985-987, (2012)

[15] R. Salvado, C. Loss, Gon, and P. Pinho, Sens., 12, 1584115857, (2012)

[16] F. Declercq, H. Rogier, and C. Hertleer, IEEE Trans. Ant. Propag., 58. (2008)

[17] T. Blecha, R. Linhart, and J. Reboun, Proc. 5th Electron. Syst. Technol. Conf. ESTC, 14-17, (2014)

[18] C. A. Balanis, 4 ed. Wiley Pub. 4. (2016) 